\title{
Complementary analyses of concrete characteristics performed on cores taken from concrete pavements
}

\author{
Aleš Frýbort ${ }^{1}$, Jana Štulířová ${ }^{1}$, and Jiř̌ Grošek ${ }^{1, *}$ \\ ${ }^{1}$ CDV - Transport Research Centre, Transport Infrastructure Department, Líšeňská 33a, 63600 Brno, \\ Czech Republic
}

\begin{abstract}
The basic assessment of cement concrete pavement condition is performed by a visual inspection, when the surface faults which are macroscopically distinguishable are documented. Detailed data on concrete condition require further tests performed on cores. Apart from common standard tests, such as layer thickness, compressive and tensile strength, resistance against water and chemical defrosting agents, other macroscopic and microscopic analyses have become useful. Some of the most important ones include petrographic assessment of aggregates (based on the fraction and type of rocks), quantification of cracks (expressed by e.g. cracking index or damage rating index), analyses of air void characteristics in concrete (expressed by e.g. spacing factor test), indication of type and extent of expansion reactions with the help of chemical reagents that visually mark the affected concrete parts. The information can be complemented and adjusted through a suitable combination of microscopic methods. Prepared thin sections are used to determine types of aggregates including the specification of its harmfulness. In combination with concrete fragments taken at least from three height levels of the core (surface-centre-bottom), differences or conformity of concrete porousness, and level and type of mineralization of pores and cracks created by secondary phases, are determined. The identification of created phases and their correlation with applied aggregates can be used for prediction of potential risks. The paper describes additional test methods that can be performed on cores from concrete structures and that are based on macroscopic and microscopic analyses.
\end{abstract}

\section{Introduction}

Extensive construction and reconstruction of the motorway and road infrastructure has been in progress in the Czech Republic in recent years. The related testing of cement concrete road pavements in operation are performed, including standard tests that describe concrete conditions. Common standard tests based on national regulations and standards are performed (layer thickness, concrete compressive and tensile strength, freeze-thaw

\footnotetext{
* Corresponding author: jiri.grosek@cdv.cz
} 
resistance) [1]. However, in some cases these tests are not sufficiently representative and macroscopic and microscopic analyses need to be made.

In cooperation with the Road and Motorway Directorate, assessments of cores taken from cement concrete motorway pavements were performed. The aim of the assessments was mainly to find causes of faults while paying attention to the risks of occurrence and development of expansive reactions in older cement concrete pavements while using new findings from abroad.

Expansive reactions in cement concrete pavements are based on a series of chemical processes accompanied with volume changes of the newly created products. Their occurrence is accompanied with micro cracks in concrete which cluster into characteristic map cracking. The expansive reactions in concrete (specifically ASR, alkali-silica reaction) was first identified and reported by T. Stanton [2]. In 1988, a group was set up within RILEM [3] to develop accelerated tests to evaluate aggregates for the risk of expansive reactions. Other groups followed, particularly TC 219-ACS [4-5].

In the evaluation of aggregates and concrete mixtures, the standard is set by dilatometric tests performed on mortar bars and concrete prisms, including the petrographic evaluation of the used aggregates. Individual testing procedures are also defined in ASTM standards [6] and national regulations. The evaluation of taken cores in relation to expansive reactions occurrence is performed with the use of orientation tests (e.g. uranyl-acetate method) or with the use of a detailed microanalysis. Cores can also be analyzed petrographically (e.g. according to ASTM C856-14 [7]).

Optical microscopy and image petrographic analysis seem to be the methods that contribute significantly to clarify the issue of ASR [8-10]. They are methods that allow to study aggregates (determine the structure, mineral composition, deformation characteristic, etc.) and at the same time observe its relationship to cement and mortar environment, or to alkali-silica gels, respectively.

For suitable techniques determining the products of expansive reactions is considered to be the study thin sections of concrete with the use of a petrographic optical microscope and the analysis of concrete fragments with the use of a scanning electron microscope - SEM, in combination with energy-dispersive X-ray spectroscopy-EDX (ASTM C1723-16 [11]).

The text below describes additional analyses that can be performed on cores from concrete structures and that are based on macroscopic and microscopic analyses.

\section{Indexes describing damage}

One of the basic options how to assess a core condition is a detailed macroscopic analysis of the core surface or its cross-section surface. Various indexes are used for comparisons of results. Two of them are specified below.

\subsection{Cracking index}

CI expresses the total width of cracks that intersect monitored lines spreading in different directions and covering a defined area. The CI unit is $\mathrm{mm} / \mathrm{m}$ [6]. CI allows continuous monitoring of fault development in road pavements, bridge pillars, concrete crash barriers, etc.

Regarding cores, this assessment procedure was complemented with a record of vertically and horizontally oriented cracks separately for the upper and lower part of the core (1/4 of height) and the central part of the core ( $1 / 2$ of height). This procedure allows determining conditions of cores in three height levels. 
Image documentation is made at the same time - surface, bottom area and core surface (assembled from eight images). The images unfold into a cylindrical area which not only shows cracks, voids, pores, etc., but also aggregates distribution (Fig. 1).
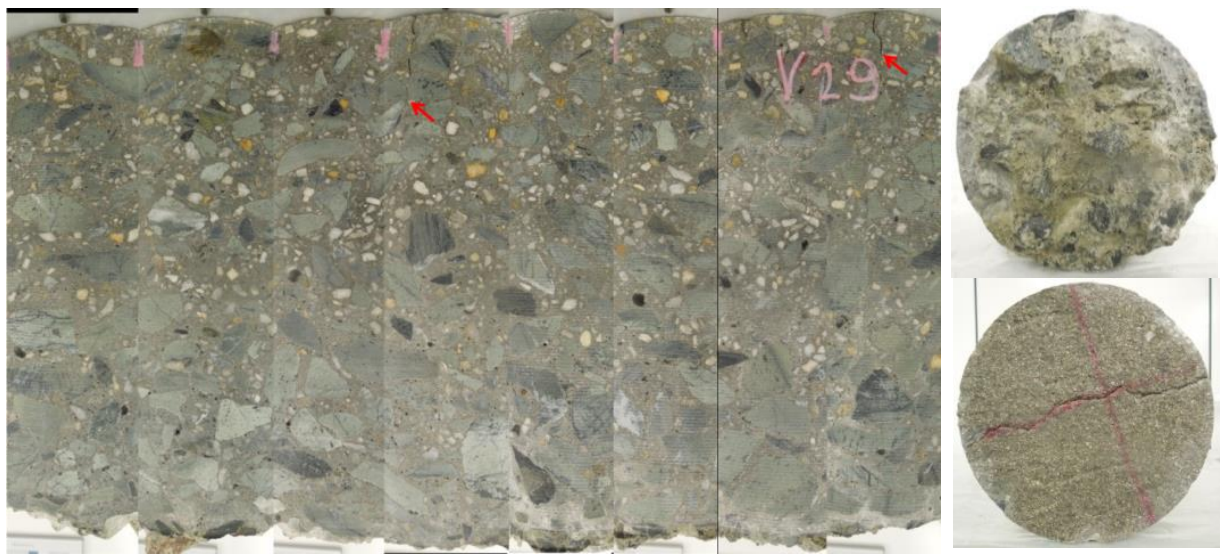

Fig. 1. Record of unfolded cylindrical area; crack width of $1 \mathrm{~mm}$ spreads just from surface to depth max. $45 \mathrm{~mm}$, CI for vertically oriented cracks in upper part of core $=3.2 \mathrm{~mm} / \mathrm{m}$.

\subsection{Damage rating index}

Damage rating index - DRI is particularly used in relation to concrete expansive reactions. The occurrence of monitored parameters for an individual $\mathrm{cm}^{2}$ (in this case $10 \times 20 \mathrm{~cm}$, see Fig. 2) is recorded from longitudinal cross-section surface of the core. Subsequently, the amount of monitored parameters occurrence is multiplied by their weight and are summed. Below are mentioned individual monitored parameters and their weights according to [6]:

- Crack in the coarse aggregate particles / 0.75

- Open crack in coarse aggregates / 4.0

- Crack with reaction product in the coarse aggregate particles / 2.0

- Coarse aggregate debonded / 3.0

- Reaction rims around aggregate particles / 0.5

- Crack in the cement paste / 2.0

- Crack with reaction product in the cement paste / 4.0

- Air voids lined or filled with reaction products / 0.5.

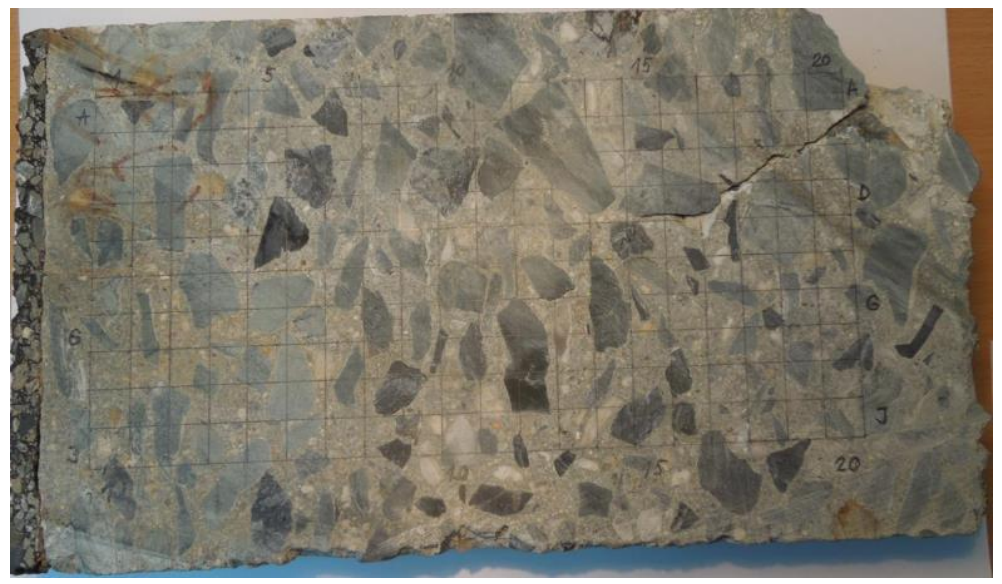


Fig. 2. Core cross-section with a matrix $10 \times 20 \mathrm{~cm}$ for calculation of DRI crack detail; in this case $\mathrm{DRI}=47$.

\section{Identification of alkali silica gels}

Preliminary identification of alkali silica gels (ASG) can be performed on the core surface or on its transversal or longitudinal cross-section. In order to ensure a quick indicative identification, chemical reagents are applied to a selected surface (fresh fracture surface), where the area with alkali silica gels is enhanced (semi quantitative method).

The most effective seems to be the result of reactions of two reagents: sodium hexanitrocobaltate and rhodamine $\mathrm{B}$. At first sodium hexanitrocobaltate $\mathrm{Na}_{3} \mathrm{Co}\left(\mathrm{NO}_{2}\right)_{6}$ is applied, which reacts with potassium in the gel producing a yellow, water insoluble, compound of potassium sodium hexanitrocobaltate $\mathrm{K}_{2} \mathrm{NaCo}\left(\mathrm{NO}_{2}\right)_{6} \cdot 6 \mathrm{H}_{2} \mathrm{O}$. Yellow spots appear at places with the presence of $\mathrm{K}^{+}$ions. Subsequently, rhodamine B is applied for qualitative identification of the calcium content in silica gels. The resulting colour shade depends on the content of elements $\mathrm{K}, \mathrm{Na}$ and $\mathrm{Ca}$. Higher content of $\mathrm{Ca}$ in the gel changes yellow orange colour into various shades of light brick red up to light purple red, see Fig. 3.

When assessing the images, it is necessary to take into account other influences that may have an impact on correct interpretation of results, e.g. types of the present aggregates. The detailed method description is specified in US patent of 1998 [12].
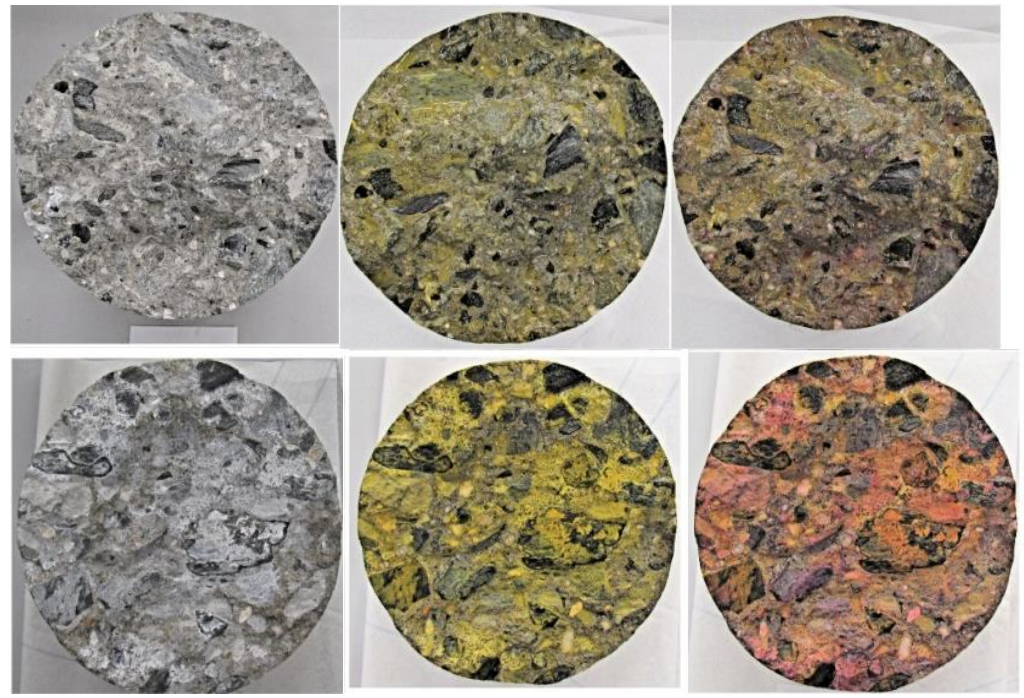

Fig. 3. Images of two cores before and after application of sodium hexanitrocobaltate (middle) and rhodamine B (right) reagents on the surface after performed tensile test; lower core is found with a high probability of silica gel occurrence.

Another method that can be used is a uranyl acetate method, which is described in Annex $\mathrm{X} 1$ of US standard ASTM C856-14 [7]. The solution of uranyl acetate $\left(\mathrm{UO}_{2}\left(\mathrm{CH}_{3} \mathrm{COO}\right)_{2}\right)$ in acetic acid is applied to the concrete surface, where sorption of $\mathrm{UO}_{2}{ }^{2+}$ occurs on negatively charged surface of the alkali silica gel. The used compound contains a radioactive isotope of uranium. The presence of the gel can best be proven by subsequent UV radiation. In this case sodium $\mathrm{Na}^{+}$ions identification is concerned. 
The ASTM standard stresses that such determined ASGs must be complemented with other methods, e.g. optical or electron microscopy. Some aggregates are even naturally luminescent under a UV lamp. Their presence must be taken into account for the assessment.

\section{Aggregates identification}

Petrographic characteristics of rocks can be either qualitative, or, if necessary, very detailed quantitative. A longitudinal cross-section through the core where it is possible to observe the distribution of aggregates and identify its types, see Fig. 4, is suitable for qualitative determination of coarse aggregates.

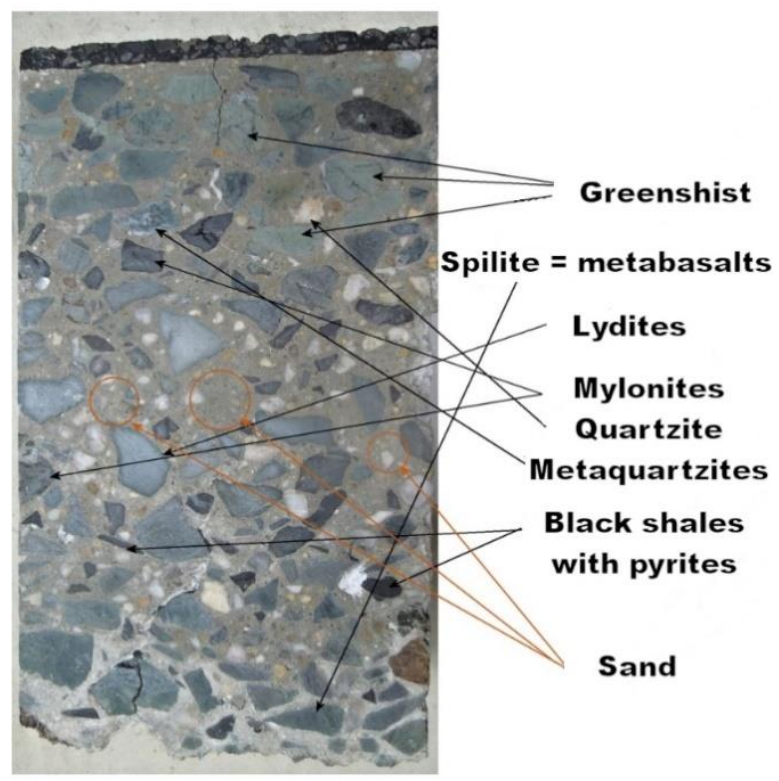

Fig. 4. Example of macroscopic identification of coarse aggregates in core cross-section.

This analysis must be specified by micro petrographic analyses of three (or more) polished thin sections (dimensions $2 \times 3 \mathrm{~cm}$ ). The classification of rock clasts of coarse aggregates is quantified with the use of petrographic polarizing microscope, which is equipped with an analyser and a polarizer for passing and reflecting light, see Fig. 5. In order to obtain more accurate results, thin sections from three height levels of the core, which represents the installed concrete segment using the same concrete recipe (of the same aggregates sources).

The determined volume percentages of minerals and rocks still need to be understood as semi quantitative indicators. Based on publications, e.g. [4] reactive aggregates are considered fragments of volcanic glass, chalcedone, opal, microcrystalline and cryptocrystalline quartz, tectonically affected quartz, and fragments of rocks that these reactive forms of $\mathrm{SiO}_{2}$ contain (e.g. silicites, lydites, cherts, quartzites - see Fig. 5, metaquartzites, mylonites, cataclasite, phyllites, etc.). The results of a detailed macro petrographic and macro petrographic analysis include average composition of the aggregates of studied samples, dividing the ratio of potentially reactive aggregates into alkali silicate reaction and into secondary sulphate expansion. 
Micro petrographic analysis allows, among others, to determine secondary sulphates, and confirms the presence of alkali silica gels and other newly produced phases in the concrete structure, see Chapter 6. In cases where we know the concrete recipe, it is possible to confirm or refute the presence of certain aggregates in taken concrete cores.

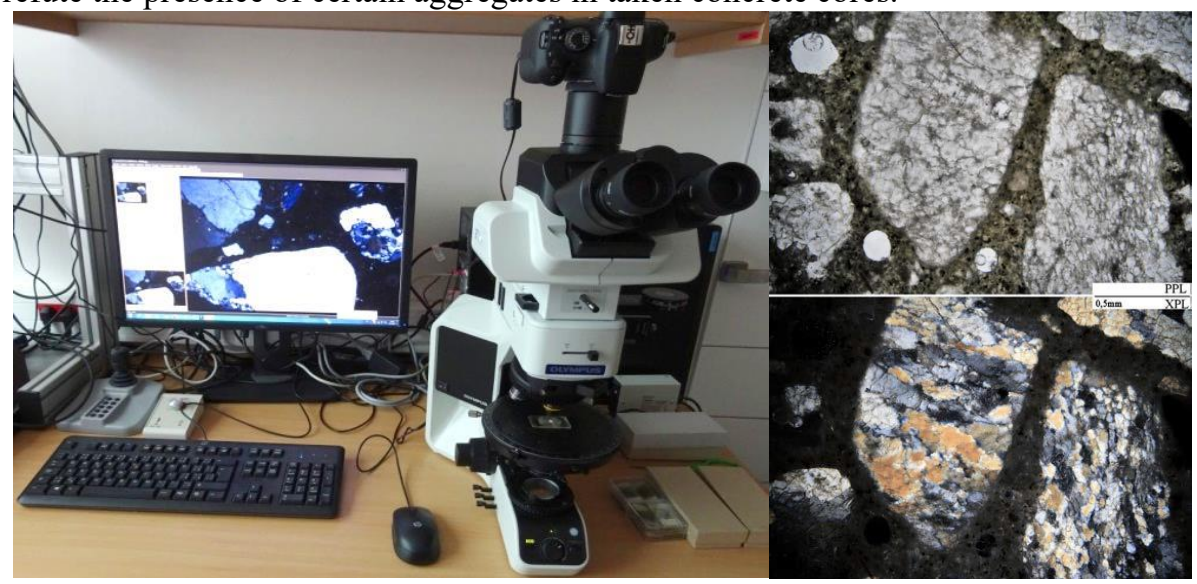

Fig. 5. Optical polarizing (petrographic) microscope Olympus BX53 (left), example of aggregates identification: quartzite fragments (right).

\section{Concrete porosity}

The amount of air pores in hardened concrete is an important parameter, which affects resistance and life span of concrete. Determination of characteristics of air pores in hardened concrete is performed according to standard ČSN EN 480-11 [13].

After performing this test, samples with dimensions of $100 \times 150 \times 20 \mathrm{~mm}$ need to be prepared, which are saw, ground flat and polished. In order to improve contrast, stamp ink colour paint is applied to a polished sample and after drying the present air pores are filled with a zinc paste. Such prepared samples are placed into a digital microscope which automatically scans the tested sample surface. It records the number of air pores intersected by measuring lines and of lengths of their chords, see Fig. 6.

This method can help to find not only the total content of air pores, but also distribution of their size, content of microscopic air pores (with diameter of $300 \mu \mathrm{m}$ and smaller) and determine the coefficient of the spatial distribution of air pores, so-called spacing factor. The spacing factor is a calculated parameter expressing maximum distance of any point in the cement sealant from the air pore edge in the cement paste.
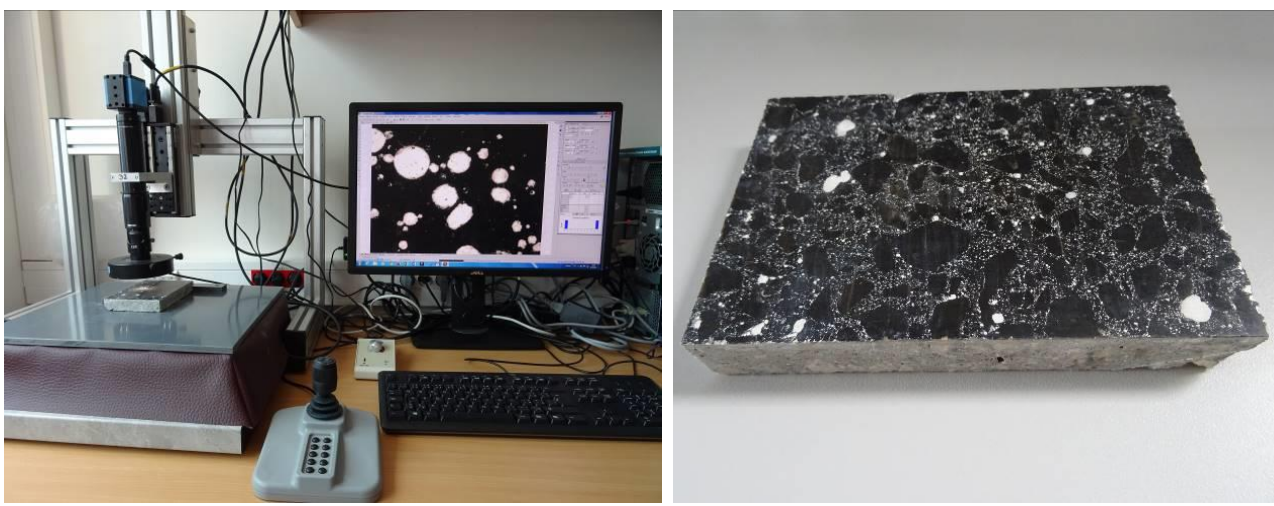
Fig. 6. Device determining characteristics of air pores in hardened concrete (left), testing sample for spacing factor parameter calculation (right).

\section{Micro chemical analyses}

The microstructure of the hardened concrete is examined with the use of a scanning electron microscope (SEM). For the need of chemical analyses this device is equipped with a probe for energy-dispersive X-ray spectroscopy (EDX), see Fig. 7. This combination of equipment is also specified in US standard ASTM C1723-16 [11].
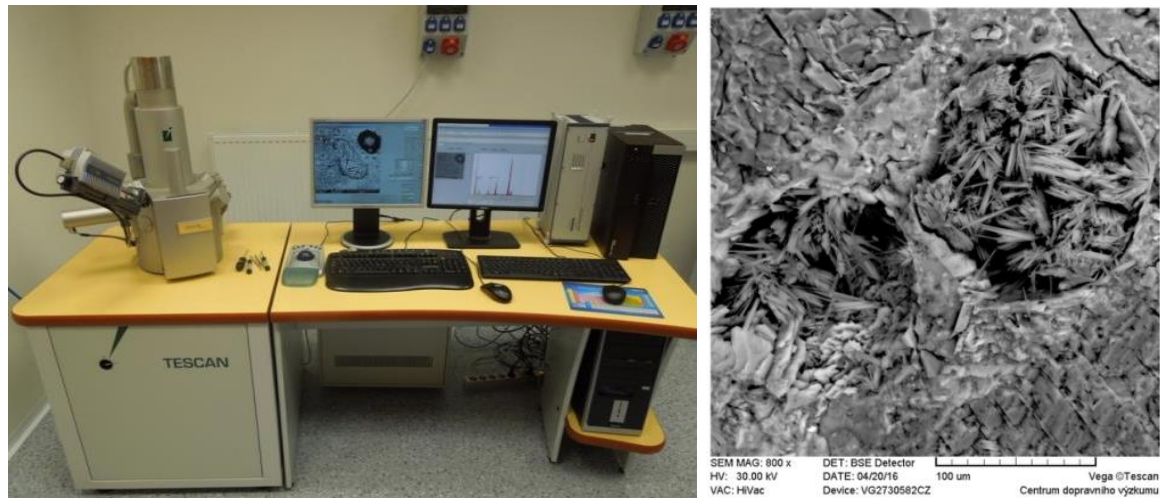

Fig. 7. Scanning electron microscope Tescan Vega equipped with a probe for energy-dispersive X-ray spectroscopy (left), image of ettringites in air voids (right).

Analyses are performed on fresh fractured surfaces of concrete samples with the size of max. $3 \mathrm{~cm}$. Like in previous cases, it is recommended to perform observations of samples taken from different core heights, at least three.

EDX provides basic quantitative information on the material element composition. There is an option to select several procedures: analysis in a selected point, line or area.

Point analysis (detection min. $5 \times 5 \mu \mathrm{m}$ ) allows performing mineral phase analysis in selected locations of studied samples (e.g. edge $\mathrm{x}$ centre).

The line scan is used for monitoring characteristic changes of the chemical composition in the present phases and of studying migration of elements in a selected cross-section. The profile change - continuous change of selected elements along a line - documents gradual development or composition change over time. It allows to determine borders between alkali silica gels and the binder, or a chemical border between aggregates and the gel (Fig. 8). 


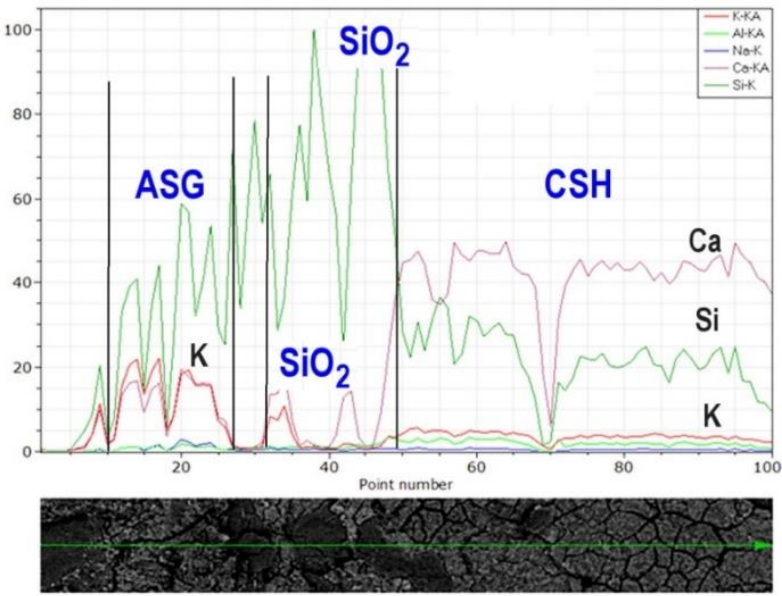

Fig. 8. Line scan documenting interface between quartz grains, CSH and ASG.

Element maps or surface distribution of elements are applied for display of chemical composition change in a selected surface. The amount of an element is expressed in so-called false colours, see Fig. 9.

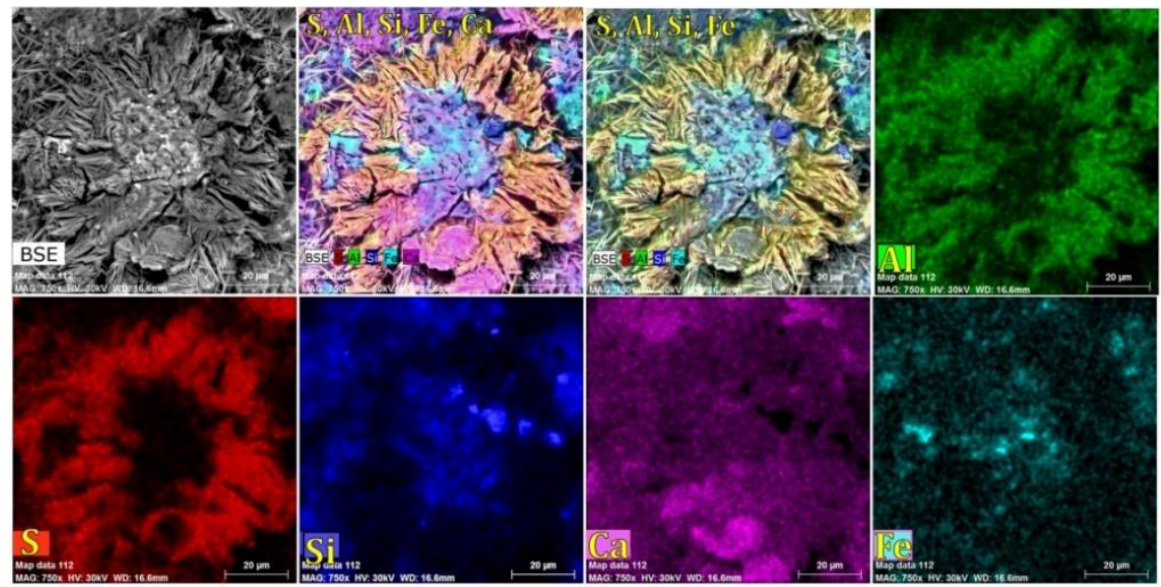

Fig. 9. Surface distribution of $\mathrm{Al}, \mathrm{Ca} . \mathrm{S}, \mathrm{Ca}$ and $\mathrm{Fe}$ in secondary ettringite sulphate.

The combination of SEM-EDX (geochemical analysis) provides the most reliable results for occurrence determination of:

- alkali silica gels and their crystalline phases,

- secondary sulphate expansion (occurrence of ettringite and thaumasite), see Fig. 7,

- portlandite in lower and upper parts of cores,

- concrete carbonization along the core height, etc.

Using an SEM helps us to find the amount of pores/capillaries filling by these products in relation to occurrence of expansion/contraction microcracks, which the products generate. With the use of an EDX it is possible to determine, for example, percentage content of $\mathrm{K}_{2} \mathrm{O}$, $\mathrm{Na}_{2} \mathrm{O}, \mathrm{SO}_{3}, \mathrm{Cl}^{-}$, etc. as well as main oxides in Portland cement clinker relics. The analyses results can be documented by a graphic record of reflective peaks, chemical composition table (Fig. 10) and images of scanned positions (Fig. 11). The identification of the degradation product can be made in a chipped concrete sample within minutes. 


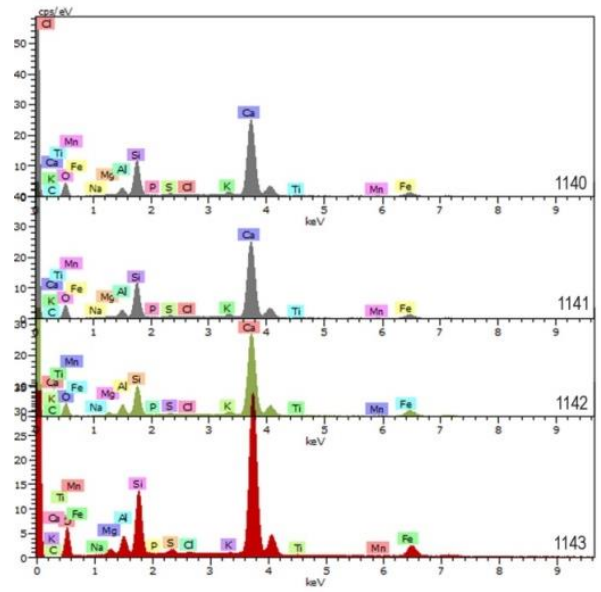

\begin{tabular}{|l|c|c|c|c|}
\hline An. No. & 1140 & 1141 & 1142 & 1143 \\
\hline \multicolumn{5}{|c|}{ w. \%] } \\
\hline $\mathrm{SiO}_{2}$ & 21.5 & 19.7 & 18.1 & 14.4 \\
\hline $\mathrm{TiO}_{2}$ & 0.1 & 0.1 & 0.5 & 0.3 \\
\hline $\mathrm{Al}_{2} \mathrm{O}_{3}$ & 3.4 & 4.1 & 6.4 & 3.7 \\
\hline $\mathrm{FeQ}$ & 2.7 & 3.2 & 4.9 & 4.2 \\
\hline $\mathrm{MnQ}$ & 0.3 & 0.3 & 0.4 & 0.4 \\
\hline $\mathrm{MgQ}$ & 1.2 & 0.9 & 2.1 & 1.0 \\
\hline $\mathrm{CaQ}$ & 43.1 & 40.2 & 42.8 & 37.5 \\
\hline $\mathrm{Na}_{2} \mathrm{O}$ & 0.6 & 0.6 & 0.5 & 0.0 \\
\hline $\mathrm{K}_{2} \mathrm{O}$ & 0.8 & 0.2 & 0.2 & 0.2 \\
\hline $\mathrm{SO}_{3}$ & 0.5 & 0.6 & 0.8 & 0.8 \\
\hline $\mathrm{Cl}_{3}$ & 0.1 & 0.0 & 0.1 & 0.1 \\
\hline $\mathrm{P}_{2} \mathrm{O}_{5}$ & 0.0 & 0.0 & 0.0 & 0.0 \\
\hline & 74.1 & 69.8 & 76.7 & 62.6 \\
\hline
\end{tabular}

Fig. 10. Example of graphic record of EDX surface analysis and chemical composition of a Portland cement clinker relic in four selected concrete positions.

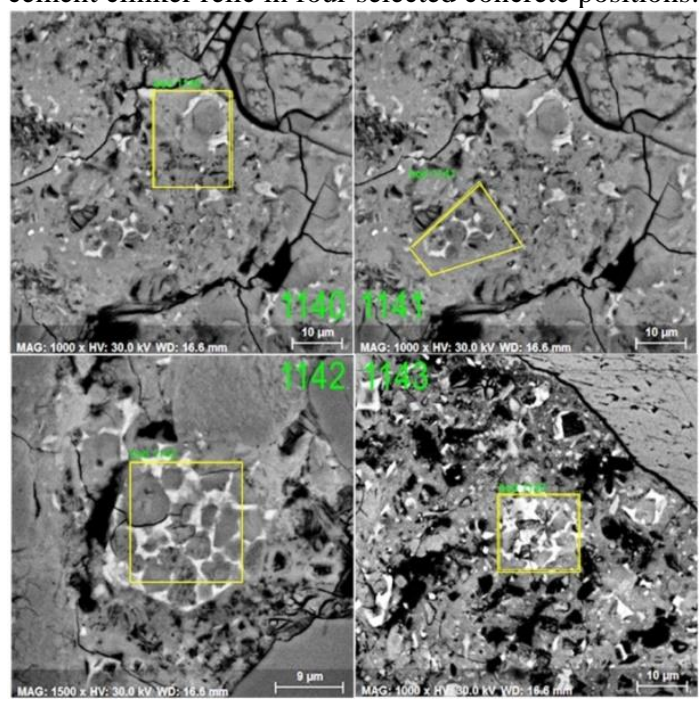

Fig. 11. Chemical analyses from Portland clinker relic surface.

It is possible, with the use of another device, to perform other more detailed analyses which are focused on a specific issue [14].

\section{Conclusions}

Transport Research Center performed an assessment of cores from cement concrete pavements in 2017 and 2018.

The analyzed cores were collected on various sections of the motorways D1, D5 and D35. The cores provided by the Road and Motorway Directorate of the Czech Republic was preceded by the evaluation of road sections with cement concrete pavement based on visual surface assessment, especially with consideration of the crack occurrence. These sections were classified in grades 0 , I, II and III. The cores were taken from boards with different degrees of classification. There were collected 3 bores, one for transverse tensile test, one for 
plain tensile test and one for detailed petrographic and microchemical analyzes. An overview of basic information from studied cores is shown in Tab. 1

Table 1. Information summary cores from cement concrete pavements

\begin{tabular}{|l|l|l|l|c|}
\hline $\begin{array}{l}\text { Motorway } \\
\text { No. }\end{array}$ & Year & $\begin{array}{l}\text { Cores } \\
\text { No. }\end{array}$ & $\begin{array}{l}\text { Transverse Tensile } \\
\text { Strength (MPa) } \\
\text { min. / avrg. / max. }\end{array}$ & $\begin{array}{l}\text { Plain Tensile } \\
\text { Strength (MPa) } \\
\text { min. / avrg. / max. }\end{array}$ \\
\hline D1 & $2004-2009$ & 32 & $3.8 / 4.6 / 5.7$ & $1.3 / 1.7 / 2.8$ \\
\hline D5 & $1996-1997$ & 25 & $3.1 / 4.7 / 5.3$ & $0.5 / 1.5 / 2.5$ \\
\hline D35 & $1995-2008$ & 18 & $3.7 / 4.5 / 6.2$ & - \\
\hline
\end{tabular}

The cores where we expected the greatest potential for the occurrence of expansive reactions were selected for petrographic and microchemical analyzes.

Motorway D1:

- The study of 9 selected boreholes did not show any significant damage by expansive reactions (ASR or degradation by secondary sulphates).

- $\quad$ Potentially hazardous aggregates: ASR approx. $11 \%$, SRR $0.5 \%$.

- Despite the fact that the middle parts of the boreholes contain sulphates, their volume is very low.

\section{Motorway D5:}

- The study carried out on 10 selected cores showed in six cases damage of ASR and SSR products.

- Potentially hazardous aggregates: ASR 6-12\%, SRR 11-18\%.

- Alkaline silica gel (ASG) varies not only between cores but also within a one core itself.

- The middle parts of the cores usually have the highest sulphate content, but at the same time there is enough free space in the air pores.

- The ASGs were recrystallized in 3 cores in the highly potassium, morphologically variable phase.

\section{Motorway D35:}

- The study of 6 cores revealed the presence of both secondary sulphates and alkaline silica gels and their crystalline phases in 2 cores.

- Potentially dangerous aggregates: ASR: up to $28 \%$ of tectonically deformed quartz, SRR not taken into account in petrographic characteristics.

- Alkaline silica gels and newly formed crystalline phases were not identified for the remaining 4 cores boreholes, even though the proportion of hazardous aggregates with so-called reactive $\mathrm{SiO} 2$ was almost identical in all boreholes.

It is noted that the conclusions based on the assessment of surface cracks (grades 0 -III) is appropriate to supplement the detailed analysis at the cores. In many cases, the study showed another type of degradation fundamentally different from assumptions. Surface cracks often extend only to a depth of $1-3 \mathrm{~cm}$ from the surface and are not directly related to the expansion reactions. It is apparent from the performed analyses that ASR and SRR are multi-step processes that are influenced by the surrounding geochemical environment and often run in parallel. 
The petrographic analysis of aggregates used in concrete is of greater importance during the assessment of the proposed concrete formula, but it can also be done additionally and correlate these results with the occurring concrete failures caused by the expansion reactions. SEM-EDX analysis is recommended for detecting the occurrence of expansive reactions and quantifying their products in concrete. It is not necessary to drill special cores for this purpose, but you can use cores remnants foe example after strength tests.

This article was produced with the financial support of the Ministry of Transport within the programme of long-term conceptual development of research institutions on the research infrastructure acquired from the Operation Programme Research and Development for Innovations (CZ.1.05/2.1.00/03.0064).

\section{References}

1. ČSN EN 206+A1: Concrete - Part 1: Specification, performance, production and conformity, (2017)

2. T.E. Stanton, O.J. Porter, L.C. Meder, A. Nicol, Proc. Am. Soc. Civ. Eng. 66, 10, 1781-1811 (1940)

3. P. Nixon, I. Sims, Mater. Struct. 29, 6, 323-334 (1996)

4. I. Fernandes, M.D.A. Ribeiro, M.A.T.M. Broekmans, I. Sims, Petrographic Atlas: Characterisation of Aggregates Regarding Potential Reactivity to Alkalis, Springer, 198p. (2016)

5. P. Nixon, I. Sims, RILEM Recommendations for the Prevention of Damage by Alkali-Aggregate Reactions in New Concrete Structures - State-of-the-Art Report of the RILEM TC 219-ACS, Springer, (2016)

6. M.D.A. Thomas, B. Fournier, K.J. Folliard, Alkali-Aggregate Reactivity (AAR) Facts Book. Report FHWA-HIF-13-019, 211p. (2013)

7. ASTM C856-14: Standard Practice for Petrographic Examination of Hardened Concrete, (2014)

8. M.A.T.M. Broekmans, Mater. Charact. 53, 129-140 (2004)

9. I. Fernandes, F. Noronha, M. Teles, Mater. Charact. 58, 1160-1170 (2007)

10. B. Fournier, M.A. Berube, K.J. Folliard, M.D.A. Thomas, Report on the diagnosis, prognosis and mitigation of alkali-silica reaction (ASR) in transportation structures, Federal Highway Administration, FHWA-HIF-09-004 (2010)

11. ASTM C1723-16: Standard Guide for Examination of Hardened Concrete Using Scanning Electron Microscopy (2016)

12. G.D. Guthrie, Jr., J.W. Carey, Detection of Alkali-Silica Reaction Swelling in Concrete by Staining, United States Patent, http://www.osti.gov/scitech/servlets/purl/871471, (1998)

13. ČSN EN 480-11: Admixtures for concrete, mortar and grout - test methods - Part 11: Determination of air void characteristics in hardened concrete, (2006)

14. A. Frýbort, D. Všianský, J. Štulírová, J. Stryk, M. Gregerová, Mater. Charact. 137, 91-108 (2018) 